\title{
DENTING POINTS IN TENSOR PRODUCTS OF BANACH SPACES
}

\author{
DIRK WERNER
}

\begin{abstract}
Let dent $A$ denote the set of denting points of a subset $A$ of some Banach space. We prove

$\operatorname{dent} \operatorname{cl} \operatorname{co}(K \otimes L)=\operatorname{dent} K \otimes \operatorname{dent} L$

for closed, bounded, absolutely convex subsets $K$ and $L$ of Banach spaces $X$ and $Y$. Here the closure refers to the completion of $X \otimes Y$ w.r.t. some reasonable crossnorm.
\end{abstract}

1. Introduction. In this note we consider denting points in subsets of tensor products of Banach spaces. Let us recall that $x$ is a denting point of a subset $K$ of a Banach space $X\left(x \in \operatorname{dent} K\right.$ for short ) if $x \notin \operatorname{cl} \operatorname{co}\left(K \backslash B_{\varepsilon}(x)\right)$ for all $\varepsilon>0$. Here $B_{\varepsilon}(x)$ denotes the closed ball with radius $\varepsilon$ centered at $x$, we write $B_{X}$ for the closed unit ball of $X$. The reader who is interested in a discussion of the relevance of denting points in connection with the Radon-Nikodým property (RNP) is referred to the monographs [2 and $\mathbf{3}$ ].

Further, recall that a norm $\alpha$ on the algebraic tensor product $X \otimes Y$ of two Banach spaces is called reasonable if $\|x \otimes y\|=\|x\| \cdot\|y\|$ for all $x \in X, y \in Y$, and if $x^{*} \otimes y^{*}$, considered as a functional on the $\alpha$-normed tensor product of $X$ and $Y$, is continuous with norm $\left\|x^{*}\right\| \cdot\left\|y^{*}\right\|$ for all $x^{*} \in X^{*}, y^{*} \in Y^{*}$. The completed $\alpha$-normed tensor product is denoted by $X \hat{\otimes}_{\alpha} Y$. ([3, Chapter VIII] is a good source for the prerequisites on tensor products which are needed.)

The main result is as follows.

THEOREM 1. Let $K$ and $L$ be closed, bounded, absolutely convex subsets of Banach spaces $X$ and $Y$, and let $\alpha$ be a reasonable crossnorm. Then

$$
\operatorname{dent} \operatorname{cl} \operatorname{co}(K \otimes L)=\operatorname{dent} K \otimes \operatorname{dent} L,
$$

where the closure refers to $X \hat{\otimes}_{\alpha} Y$ and $K \otimes L=\{x \otimes y: x \in K, y \in L\}$.

2. Proof of Theorem 1. We need two preparatory lemmas for the proof. In the following discussion let $K$ be a closed, bounded, absolutely convex subset of some real Banach space $X$, and let $x_{0} \in \operatorname{dent} K$. For $\varepsilon>0$ define $K_{\varepsilon}:=$ $\operatorname{cl} \operatorname{co}\left(K \backslash B_{\varepsilon}\left(x_{0}\right)\right)$. Using the Hahn-Banach theorem it is possible to find a functional $x^{*} \in X^{*}$ and a number $\delta>0$ (both dependent on $\varepsilon$ in general) such that $x^{*}\left(x_{0}\right)=1$ and $x^{*}(x) \leq 1-\delta$ for all $x \in K_{\varepsilon}$. It will be convenient to use the notations $K_{0}:=K$ and $\Phi_{\varepsilon}(\varepsilon \geq 0)$ for the real-valued norm-continuous function defined by $\Phi_{\varepsilon}\left(x^{*}\right):=\sup \left\{x^{*}(x): x \in K_{\varepsilon}\right\}$. The next lemma shows that it is possible to choose a separating functional as above with $\Phi_{0}\left(x^{*}\right)$ small.

Received by the editors January 7, 1986 and, in revised form, June 4, 1986.

1980 Mathematics Subject Classification (1985 Revision). Primary 46M05; Secondary 46B20.

Key words and phrases. Denting point, tensor product. 
LEMMA 2. For $x_{0} \in \operatorname{dent} K$ and $\varepsilon>0$ there exist $\delta>0$ and $x^{*} \in X^{*}$ such that

(i) $x^{*}\left(x_{0}\right)=1$,

(ii) $\Phi_{\varepsilon}\left(x^{*}\right)=1-\delta$,

(iii) $\Phi_{0}\left(x^{*}\right) \leq 1+\varepsilon \delta$.

PROOF. Choose a positive number $\alpha$ so small that $\alpha /(1-\alpha)<\varepsilon$, and choose $\beta$ to satisfy $0<\beta<\varepsilon \alpha / 2$. Separate $x_{0}$ strictly from $K_{\beta}$ by means of a functional $x^{*}$. Of course, we may assume $x^{*}\left(x_{0}\right)=1$ and $x^{*}(x)<1$ for $x \in K_{\beta}$, since $K$ is absolutely convex. Define $\delta$ by $\delta:=1-\Phi_{\varepsilon}\left(x^{*}\right)$. As $x^{*}$ was supposed to separate strictly, we have $\delta>0$. It is left to estimate $\Phi_{0}\left(x^{*}\right)$, that is $x^{*}(x)$ for $x \in K$.

It is enough to do so for $x \in K \cap B_{\beta}\left(x_{0}\right)$, the remaining $x$ 's satisfy $x^{*}(x) \leq 1$ anyway. Now consider an arbitrary $y \in K$ with $\left\|y-x_{0}\right\|>\varepsilon$. Then

$$
\left\|\alpha y+(1-\alpha) x-x_{0}\right\|>\beta \text {. }
$$

In fact, otherwise we would have (taking into account $\left\|x-x_{0}\right\| \leq \beta$ )

$$
\begin{aligned}
\left\|\alpha\left(y-x_{0}\right)\right\| & \leq\left\|\alpha y+(1-\alpha) x-x_{0}\right\|+\left\|(1-\alpha)\left(x-x_{0}\right)\right\| \\
& \leq \beta+(1-\alpha) \beta<\alpha \varepsilon,
\end{aligned}
$$

which contradicts the choice of $y$. Hence,

$$
x^{*}(\alpha y+(1-\alpha) x) \leq 1 .
$$

Taking the supremum over all $y \in K \backslash B_{\varepsilon}\left(x_{0}\right)$, which amounts to the same as the supremum over all $y \in K_{\varepsilon}$, we obtain

$$
\alpha \Phi_{\varepsilon}\left(x^{*}\right)+(1-\alpha) x^{*}(x) \leq 1
$$

and thus $x^{*}(x) \leq 1+\varepsilon \delta$ by the choice of $\delta$ and $\alpha$.

We need a stronger version of Lemma 2 . Note that we have actually proved the following statement: Let $\beta$ be as in the above proof, and let

$$
C:=\left\{x^{*} \in X^{*}: x^{*}\left(x_{0}\right)=1, \Phi_{\beta}\left(x^{*}\right) \leq 1\right\} .
$$

Then $\Phi_{0}\left(x^{*}\right) \leq 1+\varepsilon \delta$ for $x^{*} \in C$ and $\delta:=1-\Phi_{\varepsilon}\left(x^{*}\right)$.

LEMMA 3. For $x_{0} \in \operatorname{dent} K$ and $\varepsilon>0$ there exists $\delta_{0}>0$ such that for all $0<\delta \leq \delta_{0}$ there is $x^{*} \in X^{*}$ with

(i) $x^{*}\left(x_{0}\right)=1$.

(ii) If $x^{*}(x)>1-\delta$ and $x \in K$, then $\left\|x-x_{0}\right\| \leq \varepsilon$.

(iii) $x^{*}(x) \leq 1+\varepsilon \delta$ for all $x \in K$.

PROOF. The second condition is nothing but $\Phi_{\varepsilon}\left(x^{*}\right) \leq 1-\delta$. Let $\beta$ and $C$ be as above, and let $\eta:=\inf \left\{1-\Phi_{\varepsilon}\left(x^{*}\right): x^{*} \in C\right\}$.

First case: $\eta>0$. Put $\delta_{0}:=\eta$. Given $0<\delta \leq \delta_{0}$ consider $\varepsilon^{*}=\min (\beta, \varepsilon \delta)$. Lemma 2 (with $\varepsilon^{*}$ instead of $\varepsilon$ ) yields $x^{*} \in X^{*}$ and $\delta^{*}>0$ with $x^{*}\left(x_{0}\right)=1$ and $\Phi_{\beta}\left(x^{*}\right) \leq \Phi_{\varepsilon^{*}}\left(x^{*}\right) \leq 1$, hence $x^{*} \in C$. Therefore, $1-\Phi_{\varepsilon}\left(x^{*}\right) \geq \eta \geq \delta$. $x^{*}$ satisfies the third condition, too, since by Lemma 2

$$
\Phi_{0}\left(x^{*}\right) \leq 1+\varepsilon^{*} \delta^{*} \leq 1+\varepsilon \delta .
$$

Second case: $\eta=0$. Choose $\delta_{0}>0$ and $x_{0}^{*} \in \mathcal{C}$ according to Lemma 2, in particular $\Phi_{\varepsilon}\left(x_{0}^{*}\right)=1-\delta_{0}$. The continuous funciton $1-\Phi_{\varepsilon}$ maps the convex set $C$ onto an interval, hence $\left.] 0, \delta_{0}\right] \subset\left(1-\Phi_{\varepsilon}\right)(C)$. In other words, for $0<\delta \leq \delta_{0}$ there 
exists $x^{*} \in \mathcal{C}$ with $\Phi_{\varepsilon}\left(x^{*}\right)=1-\delta$. According to our above remarks, $x^{*}$ fulfills the conclusions of Lemma 3 .

PROOF OF THEOREM 1. We restrict ourselves to the case of real Banach spaces, the complex case can be established along the same lines. Moreover, we assume that $K$ and $L$ are contained in the respective unit balls of $X$ and $Y$.

Let $x_{0} \in \operatorname{dent} K, y_{0} \in \operatorname{dent} L$, and $\varepsilon>0$. Using Lemma 2 we find $x^{*} \in X^{*}, y^{*} \in$ $Y^{*}, \delta_{1}>0, \delta_{2}>0$ with the properties

$$
\begin{aligned}
& x^{*}\left(x_{0}\right)=y^{*}\left(y_{0}\right)=1, \\
& x \in K \text { and } x^{*}(x)>1-\delta_{1} \text { imply }\left\|x-x_{0}\right\| \leq \varepsilon, \\
& y \in L \text { and } y^{*}(y)>1-\delta_{2} \text { imply }\left\|y-y_{0}\right\| \leq \varepsilon, \\
& x^{*}(x) \leq 1+\varepsilon \delta_{1} \text { for all } x \in K, \\
& y^{*}(y) \leq 1+\varepsilon \delta_{2} \text { for all } y \in L .
\end{aligned}
$$

Lemma 3 permits us to assume $\delta_{1}=\delta_{2}=: \delta$.

In the first step we are going to prove

$$
\begin{gathered}
x \in K, y \in L, \quad \text { and }\left\langle x^{*} \otimes y^{*}, x \otimes y\right\rangle>1-\delta / 2 \text { imply } \\
\left\|x \otimes y-x_{0} \otimes y_{0}\right\|_{\alpha} \leq 2 \varepsilon .
\end{gathered}
$$

In fact,

$$
\begin{aligned}
\delta / 2 & >\left\langle x^{*} \otimes y^{*}, x_{0} \otimes y_{0}-x \otimes y\right\rangle \\
& =\left(1-x^{*}(x)\right) y^{*}(y)+\left(1-y^{*}(y)\right) \\
& \geq(-\varepsilon \delta) y^{*}(y)+\left(1-y^{*}(y)\right) \quad\left(\text { w.l.o.g. } y^{*}(y) \geq 0\right) \\
& \geq-\varepsilon \delta(1+\varepsilon \delta)+\left(1-y^{*}(y)\right),
\end{aligned}
$$

hence

$$
1-y^{*}(y)<\delta / 2+\varepsilon \delta(1+\varepsilon \delta)<\delta
$$

for $\varepsilon$ small enough. It follows that $\left\|y-y_{0}\right\| \leq \varepsilon$. Analogously we deduce $\left\|x-x_{0}\right\| \leq \varepsilon$, and we end up with

$$
\left\|x \otimes y-x_{0} \otimes y_{0}\right\|_{\alpha} \leq\left\|x \otimes\left(y-y_{0}\right)\right\|_{\alpha}+\left\|\left(x-x_{0}\right) \otimes y_{0}\right\|_{\alpha} \leq 2 \varepsilon .
$$

In the second step we shall prove

$$
\begin{gathered}
u \in \operatorname{co}(K \otimes L) \text { and }\left\langle x^{*} \otimes y^{*}, u\right\rangle>1-\varepsilon \delta / 2 \text { imply } \\
\left\|u-x_{0} \otimes y_{0}\right\|_{\alpha} \leq 16 \varepsilon
\end{gathered}
$$

which is enough to show $x_{0} \otimes y_{0} \in \operatorname{dent} \operatorname{cl} \operatorname{co}(K \otimes L)$. To prove (2), consider a convex combination $u=\sum_{N} \lambda_{i} \cdot x_{i} \otimes y_{i} \in \operatorname{co}(K \otimes L)$, and define the following subsets of $N=\{1, \ldots, n\}$ :

$$
\begin{aligned}
& I=\left\{i \in N:\left\|x_{i} \otimes y_{i}-x_{0} \otimes y_{0}\right\|_{\alpha} \leq 2 \varepsilon\right\} \\
& J=\left\{i \in N:\left\|x_{i} \otimes y_{i}-x_{0} \otimes y_{0}\right\|_{\alpha}>2 \varepsilon\right\} \\
& J^{\prime}=\left\{i \in N:\left\langle x^{*} \otimes y^{*}, x_{i} \otimes y_{i}\right\rangle \leq 1-\delta / 2\right\} \\
& R=\left\{i \in N:\left\langle x^{*} \otimes y^{*}, x_{i} \otimes y_{i}\right\rangle>1\right\}
\end{aligned}
$$


$J \subset J^{\prime}$ by (1), therefore

$$
\begin{aligned}
\sum_{J} \lambda_{i} \leq & \sum_{J^{\prime}} \lambda_{i} \\
\leq & \frac{2}{\delta} \sum_{J^{\prime}} \lambda_{i} \cdot\left(1-\left\langle x^{*} \otimes y^{*}, x_{i} \otimes y_{i}\right\rangle\right) \\
\leq & \frac{2}{\delta}\left(\sum_{N}-\sum_{R}\right)\left(\lambda_{i} \cdot\left(1-\left\langle x^{*} \otimes y^{*}, x_{i} \otimes y_{i}\right\rangle\right)\right) \\
\leq & \frac{2}{\delta}\left(1-\left\langle x^{*} \otimes y^{*}, u\right\rangle\right. \\
& \left.\quad+\sum_{R} \lambda_{i} \cdot\left(\sup \left\{x^{*}(x): x \in K\right\} \cdot \sup \left\{y^{*}(y): Y \in L\right\}-1\right)\right) \\
\leq & \frac{2}{\delta}\left(\varepsilon \delta / 2+(1+\varepsilon \delta)^{2}-1\right) \leq 7 \varepsilon
\end{aligned}
$$

Consequently,

$$
\left\|u-x_{0} \otimes y_{0}\right\|_{\alpha} \leq\left(\sum_{I}+\sum_{J}\right)\left(\lambda_{i}\left\|x_{i} \otimes y_{i}-x_{0} \otimes y_{0}\right\|_{\alpha}\right) \leq 2 \varepsilon+7 \varepsilon \cdot 2=16 \varepsilon .
$$

Conversely, let $v \in \operatorname{dent} \operatorname{cl} \operatorname{co}(K \otimes L)$. Since dent $\operatorname{cl} \operatorname{co} D \subset \operatorname{cl} D$ holds for any bounded subset $D$ of any Banach space, and since $K \otimes L$ is easily seen to be closed in $X \hat{\otimes}_{\alpha} Y$, it follows that $v \in K \otimes L$. Thus, $v=x_{0} \otimes y_{0}$ for some $x_{0} \in K, y_{0} \in L$. Necessarily $x_{0}$ and $y_{0}$ have to be denting points of $K$ and $L$.

It should be pointed out that Theorem 1 does not extend to merely convex closed bounded sets $K$ or $L$. In fact, if $K$ and $L$ are closed, bounded, and convex with $K$, in addition, absolutely convex, then $M:=\operatorname{cl} \operatorname{co} K \otimes L$ is absolutely convex, too, so that (by Theorem 1) $x \otimes y \in \operatorname{dent} M$ if and only if $x \in \operatorname{dent} K$ and $y \in \operatorname{dent} \operatorname{aco} L$. Of course, the inclusion dent $M \subset \operatorname{dent} K \otimes \operatorname{dent} L$ remains valid also in the general case.

Let us draw some easy conclusions from Theorem 1. Since the unit ball of the projective tensor product is by definition $\operatorname{cl} \operatorname{co}\left(B_{X} \otimes B_{Y}\right)$ we obtain

COROLlaRY 4 . dent $B_{X \hat{\otimes}_{\pi} Y}=\operatorname{dent} B_{X} \otimes \operatorname{dent} B_{Y}$.

This result was proved in [8] in an entirely different manner. In connection with the discussion of RNP it is interesting to note that the projective tensor product of spaces with RNP need not have RNP [1]. Moreover, Theorem 1 and Corollary 4 remain true if "denting point" is replaced by "strongly exposed point" (in the latter case the separating functional may be chosed independently of $\varepsilon$ ). Thus, we recover the result of [7] which has proved useful in the study of Banach spaces, in particular spaces of operators, e.g. $[4,5,6]$.

Our final remark concerns extreme functionals on spaces of bounded linear operators (cf. also [8] for interesting results). Under the identification of the Banach space $V$ with a subsapce of $V^{* *}$ the inclusion dent $B_{V} \subset \operatorname{dent} B_{V^{* *}}$ is always valid (quickest proof by means of the principle of local reflexivity). In particular it follows 
from Corollary 4 that

$$
\begin{aligned}
\operatorname{dent} B_{X^{* *}} & \otimes \operatorname{dent} B_{Y^{* *}} \subset \operatorname{dent} B_{X^{* *}} \hat{\otimes}_{\pi} Y^{* *} \\
& \subset \operatorname{dent} B_{\left(X^{* *} \hat{\otimes}_{\pi} Y^{* *}\right)^{* *}}=\operatorname{dent} B_{L\left(X^{* *}, Y^{* * *}\right)^{*}}
\end{aligned}
$$

so that

$$
\operatorname{dent} B_{X} \otimes \operatorname{dent} B_{Y} \subset \operatorname{dent} B_{X^{* *}} \otimes \operatorname{dent} B_{Y^{* *}} \subset \operatorname{dent} B_{L\left(X, Y^{*}\right)^{*}},
$$

as $L\left(X, Y^{*}\right)^{*}$ is isometric to a subspace of $L\left(X^{* *}, Y^{* * *}\right)^{*}$ containing $X^{* *} \otimes Y^{* *}$. A similar result does not hold for extreme points. P. Harmand has found an example which shows that $T \rightarrow\left\langle T^{* *} p, q\right\rangle$ need not be an extreme functional on $L(X, Y)$, although $p \in X^{* *}$ and $q \in Y^{*}$ (or even $q \in Y^{* * *}$ ) are extreme points of the respective unit balls. On the other hand it has been observed in [9] that $T \rightarrow$ $\left\langle T \mathbf{1}, \delta_{k}\right\rangle$ is an extreme functional on $L(C K, C K)$ if $k$ is an isolated point of $K$ or $K=\beta I$ for a discrete set $I$ (the point here is that $1 \notin \operatorname{dent} B_{C K}$ unless $K$ is finite).

\section{REFERENCES}

1. J. Bourgain and G. Pisier, A construction of $\mathcal{L}_{\infty}$-spaces and related Banach spaces, Bol. Soc. Brasil. Mat. 14 (1983), 109-123.

2. R. D. Bourgin, Geometric aspects of convex sets with the Radon-Nikodym property, Lecture Notes in Math., Vol. 993, Springer-Verlag, Berlin and New York, 1983.

3. J. Diestel and J. J. Uhl, Vector measures, Math. Surveys, no. 15, Amer. Math. Soc., Providence, R.I., 1977.

4. M. Feder and P. Saphar, Spaces of compact operators and their dual spaces, Israel J. Math. 21 (1975), 28-49.

5. G. Godefroy, Nicely smooth Banach spaces, Longhorn Notes, The University of Texas at Austin Functional Analysis Seminar, 1984-1985, pp. 117-124.

6. G. Godefroy and P. Saphar, Duality in spaces of operators and smooth norms on Banach spaces (to appear).

7. S. Heinrich, Strongly exposed and conical points in a projective tensor product, Teor. Funksiı Funktsional. Anal. i Prilozhen. 22 (1975), 146-154. (Russian)

8. W. M. Ruess and C. P. Stegall, Weak*-denting points in duals of operator spaces, Banach Spaces, Proc. Missouri Conf. Columbia 1984, Lecture Notes in Math., Vol. 1166, SpringerVerlag: Berlin and New York, 1986, pp. 158-168.

9. D. Werner and W. Werner, On the $M$-structure of the operator space $L(C K)$, Preprint.

Institut für Mathematik I, Freie Universität Berlin, Arnimallee 3, D-1000 Berlin 33, Federal REPUblic OF Germany 